\title{
Giải pháp cảnh báo tránh va chạm dựa trên dữ liệu môi trường và đặc điểm điều khiển phương tiện
}

\author{
Quách Hải Thọ ${ }^{1}$, Huỳnh Công Pháp ${ }^{2}$, Phạm Anh Phương ${ }^{3}$ \\ 1 Trường Đại học Nghệ thuật, Đại học Huế \\ 2 Trường Đại học Công nghệ Thông tin và Truyền thông Việt - Hàn, Đại học Đà Nẵng \\ 3 Khoa Tin học, Trường Đại học Sư phạm, Đại học Đà Nẵng
}

Tác giả liên hệ: Quách Hải Thọ, qhaitho@hueuni.edu.vn

Ngày nhận bài: 13/04/2021, ngày sửa chữa: 01/06/2021, ngày duyệt đăng: 12/06/2021

Định danh DOI: 10.32913/mic-ict-research-vn.v2021.n1.963

Tóm tắt: Trong quá trình chuyển động của phương tiện khi tham giao thông, các yếu tố cần được xét đến là các tính năng an toàn để tạo nên sự thoải mái cho người ngồi trên xe. Trong bài báo này, với việc phân tích mối quan hệ giữa thời gian tránh va chạm kết hợp với dữ liệu thông tin môi trường, cùng với các thông số dựa trên đặc điểm của người điều khiển phương tiện, từ đó đề xuất giải pháp mô hình cảnh báo tránh va chạm cho phương tiện. Đặc điểm của mô hình cảnh báo tránh va chạm này có thể thích ứng với nhiều điều kiện điều khiển phương tiện khác nhau và đưa ra cảnh báo thích hợp. Kết quả mô phỏng được thực hiện trong môi trường mô phỏng Matlap để chứng minh giải pháp đề xuất hoạt động hiệu quả với những điểm tối ưu như giảm thiểu rủi ro va chạm và cải thiện mức độ an toàn khi điều khiển phương tiện.

Từ khóa: Thòi gian va chạm, lập quy hoạch đường đi, lập kế hoạch chuyển động, hệ thống giao thông thông minh..

Title: Solutions on Collision Avoidance based on Environmental Data and Vehicle Control Characteristics

Abstract: In the process of movement of vehicles when participating in traffic, the factors that need to be taken into account are safety features to create comfort for passen-gers and drivers. In this article, with the analysis of the relationship between time to collision combined with environmental information data, along with parameters based on the characteristics of the vehicle driver, thereby proposing a collision warning model solution for the vehicle. The characteristics of this collision warn-ing model can adapt to a variety of vehicle control conditions and give an appro-priate warning threshold. Simulation results are carried out in a Matlap simulation environment to prove that the proposed solution works efficiently with optimal points such as minimizing the risk of collisions and improving the safety level when driving vehicles.

Keywords: Time to collision, path planning, motion planning, intelligent transportation systems.

\section{GIỚI THIỆU}

Trong những năm gần đây, với nhiều kết quả nghiên cứu về bài toán cảnh báo va chạm cho phương tiện tham gia giao thông đã có thể được phân thành 2 hướng, gồm thuật toán xác định về khoảng cách an toàn [5] và thuật toán xác định về thời gian an toàn [8]. Trong đó, thuật toán xác định về thời gian an toàn sử dụng giá trị thời gian va chạm, sẽ thực hiện việc so sánh thời gian va chạm giữa 2 đối tượng với ngưỡng thời gian an toàn, để từ đó đưa ra quyết định quá trình chuyển động của xe đang ở trạng thái an toàn hay không. Nhóm thuật toán xác định về khoảng cách an toàn sẽ đề cập đến khoảng cách tránh va chạm tối thiểu giữa xe chủ và chướng ngại vật, đây cũng là khoảng cách mà xe chủ cần phải duy trì để tránh va chạm với chướng ngại vật trong điều kiện hoạt động hiện tại của xe[10].

Do hoạt động trong môi trường phức tạp, với các yếu tố ảnh hưởng đến quá trình quy hoạch chuyển động của phương tiện như: hệ thống đường giao thông, điều kiện thời tiết khác nhau, những hạn chế của hệ thống cảm biến, các tình huống bất thường do các đối tượng tham gia giao thông khác... [1-3,9], đã tạo nên sự phức tạp thay đổi theo thời gian và các tình huống không thể dự đoán trước. Do đó, các thuật toán chỉ thuần túy về xác định khoảng cách an toàn và khoản thời gian an toàn chưa đủ linh hoạt để 
thích ứng với đa số các trường hợp xảy ra [7, 12].

Một vài nghiên cứu trước đây cũng đã chỉ ra rằng, với nhiều đối tượng điều khiển phương tiện khác nhau thì khả năng phản ứng trước tình huống nguy hiểm cũng khác nhau. Như trong nghiên cứu [4], H. Zhou và các cộng sự đã chứng minh những tác động không hiệu quả của hệ thống cảnh báo khi thực hiện tín hiệu cảnh báo quá sớm đã gây ra những can thiệp xấu cho đối tượng là người điều khiển trẻ tuổi, vì với nhóm đối tượng này thì khả năng phản ứng với những hình huống nguy hiểm nhanh hơn các nhóm đối tượng lớn tuổi. Ngược lại, với nhóm đối tượng lớn tuổi thì khả năng phản ứng chậm, nếu tín hiệu cảnh báo phát ra với khoảng cách để tạo tín hiệu cảnh báo như nhóm đối tượng trẻ tuổi thì quá trình điều khiển chuyển động của nhóm đối tượng này càng trở nên nguy hiểm. Tương tự như vậy, trong các điều kiện thời tiết khác nhau, như ban ngày và ban đêm, hoặc điều kiện thời tiết có tầm nhìn khác nhau thì thời điểm đưa ra tín hiệu cảnh báo cũng không thể giống nhau.

Như vậy, khi đang điều khiển phương tiện, nếu đối tượng điều khiển phương tiện phát hiện ra vấn đề nguy hiểm cần phải thực hiện quá trình điều khiển tránh nguy hiểm thì không cần phải thu nhận tín hiệu từ hệ thống cảnh báo. Ngược lại, nếu đối tượng điều khiển không tìm thấy tình huống nguy hiểm kịp thời, thì thời gian cảnh báo tránh va chạm để đảm bảo an toàn do hệ thống cảnh báo nguy hiểm đưa ra là thời gian tối thiểu để đối tượng điều khiển phương tiện thực hiện các biện pháp nhằm đảm bảo an toàn, khoảng thời gian này bao gồm thời gian phản ứng của đối tượng điều khiển và thời gian cần thiết để điều khiển phương tiện [12], quá trình điều khiển phương tiện có thể giảm tốc độ bằng thao tác phanh hoặc thực hiện thao tác thay đổi quỹ đạo chuyển động bằng thao tác điều khiển lái.

Trong bài báo này, dựa trên giải pháp cảnh báo tránh va chạm xác định khoảng thời gian an toàn, sẽ đề xuất một giải pháp cảnh báo tránh va chạm mới, trong đó kết hợp thêm yếu tố của dữ liệu môi trường xung quanh đã thu nhận được, cùng với đặc điểm điều khiển chuyển động của xe. So với các giải pháp trước đây, giải pháp đề xuất này có khả năng thích ứng và linh hoạt trong các điều kiện phức tạp của môi trường hoạt động, điều này có thể cải thiện được hiệu quả quy hoạch chuyển động và nâng cao tính an toàn của phương tiện. Kết quả mô phỏng được tiến hành trong môi trường Matlap để kiểm chứng giải pháp đưa ra đạt được hiệu quả hoạt động, với quá trình cảnh báo tránh va chạm trong quy hoạch chuyển động của phương tiện.

Phần tiếp theo của bài báo sẽ giới thiệu một số tình huống để làm nền tảng cho việc giải quyết bài toán cảnh báo tránh va chạm đặt ra, từ đó đề xuất mô hình giải pháp để thực hiện thao tác cảnh báo tránh va chạm cho phương tiện chủ dựa trên các yếu tố về thời gian, thông tin môi trường và điều kiện hoạt động của xe. Tiếp theo là phần thực nghiệm và kết luận với một số đề xuất các hướng nghiên cứu tiếp theo cho bài toán nâng cao tính năng an toàn của phương tiện.

\section{XÂY DỰNG MÔ HÌNH CẢNH BÁO TRÁNH VA CHAM}

\section{Phân tích bài toán}

Hệ thống cảnh báo tránh va chạm đang là một trong những tiêu chuẩn được đưa vào danh sách đánh giá an toàn cho phương tiện. Những hệ thống cảnh báo tránh va chạm sử dụng các thiết bị cảm biến để thu nhận dữ liệu môi trường xung quanh, những dữ liệu này được phân tích để phát hiện ra các trường hợp nguy hiểm mà từ đó kích hoạt hệ thống phanh khẩn cấp hoặc đưa ra tín hiệu cảnh báo khẩn cấp nhằm có quyết định điều chỉnh hướng chuyển động phù hợp. Để giải quyết bài toán cảnh báo tránh va chạm, chúng ta sẽ phân tích tình huống có thể xảy ra va chạm trong quá trình hoạt động của phương tiện, gồm: tình huống va chạm trực diện hay va chạm phía trước và tình huống va chạm từ phía sau.

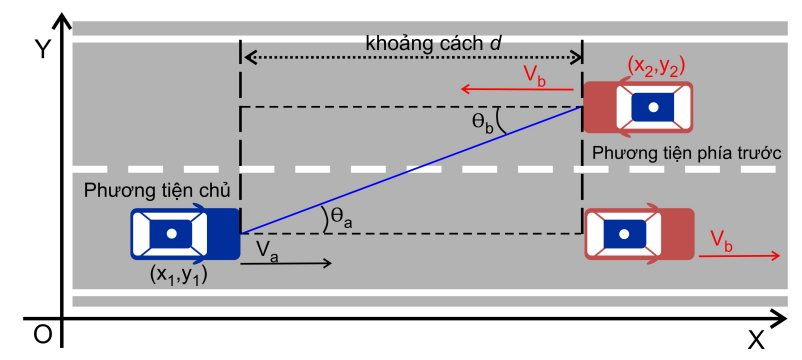

Hình 1. Minh họa tình huống tham gia giao thông

Với $V_{a}$ là vận tốc của phương tiện chủ, $V_{b}$ là vận tốc của phương tiện phía trước, $\theta_{a}$ là góc lệch hướng của phương tiện chủ và $\theta_{b}$ là góc lệch hướng của phương tiện phía trước, giá trị $\theta_{a}$ và $\theta_{b}$ được tính từ vị trí trung tâm của xe đến đường trung tâm giữa 2 xe (hình 1$), W_{a}$ là chiều rộng của phương tiện chủ và $W_{b}$ là chiều rộng của phương tiện phía trước, $k$ là giá trị trung bình chiều rộng của 2 xe được tính bằng $k=\left(W_{a}+W_{b}\right) / 2$. Vị trí của các phương tiện được xác định trong hệ trục tọa độ Descarter $(\mathrm{X}, \mathrm{Y})$, với $\left(x_{1}, y_{1}\right)$ là vị trí của phương tiện chủ, $\left(x_{2}, y_{2}\right)$ là vị trí của phương tiện phía trước, thì giá trị d là khoảng cách giữa 2 phương tiện được tính như sau:

$$
d=\sqrt{\left(x_{2}-x_{1}\right)^{2}+\left(y_{2}-y_{1}\right)^{2}}
$$

Nếu 2 xe di chuyển ngược chiều, thì tình huống xảy ra va chạm trực diện khi: 


$$
k \leq\left|d * \sin \theta_{a}\right|
$$

Và khoảng thời gian 2 xe sẽ xảy ra va chạm:

$$
t=4,3 * \frac{\left(d *\left|\cos \theta_{a}\right|-5\right)}{V_{a}+V_{b}}
$$

Khoảng thời gian xảy va chạm sẽ được tính toán khi $\left|\theta_{a}\right| \leq 90^{\circ}$ và $\left|\theta_{b}\right|>90^{\circ}$. Trường hợp ngược lại khi $\left|\theta_{a}\right| \geq$ $90^{\circ}$ và $\left|\theta_{b}\right| \leq 90^{\circ}$ thì không tính giá trị này, vì không xảy ra va chạm. Nếu 2 xe di chuyển cùng chiều, thì trường hợp xảy ra va chạm từ phía sau khi vận tốc của phương tiện chủ lớn hơn vận tốc của phương tiện phía trước $V_{a}>V_{b}$. Khoảng thời gian 2 xe sẽ xảy ra va chạm:

$$
t=\frac{d}{V_{a}+V_{b}}
$$

Qua phân tích các tình huống nêu trên, có thể thấy rằng phương pháp tính toán thời gian xảy ra va chạm là như nhau cho dù là va chạm trực diện hay và chạm từ phía sau. Do đó, để giải quyết bài toán trong giải pháp này, chúng ta sẽ sử dụng đến giá trị thời gian va chạm nhằm nâng cao khả năng thích ứng của giải pháp trong môi trường phức tạp của quá trình quy hoạch chuyển động cho phương tiện khi tham gia giao thông.

\section{Xây dựng thuật toán}

Ý tưởng chính của giải pháp này là chọn ngưỡng khoảng cách hợp lý để thực hiện cảnh báo. Việc chọn ngưỡng khoảng cách để thực hiện cảnh báo là giải pháp được đánh giá đảm bảo hiệu quả an toàn, vì nếu khoảng cách cảnh báo quá dài thì sẽ gây tác động xấu và nhiễu thông tin cho hoạt động điều khiển phương tiện, nếu khoảng cách cảnh báo quá ngắn thì hiệu quả cảnh báo tránh va chạm không tối ưu và không thể tránh được nguy hiểm. Vì vậy, giải pháp đề xuất trong nghiên cứu này được lựa chọn là ngưỡng cảnh báo phù hợp, điều này không chỉ tạo ra tác động tốt và không nhiê̂u thông tin cho hoạt động điều khiển, mà còn giúp nâng cao hiệu quả an toàn khi điều khiển phương tiện. Hơn nữa, trong các điều kiện môi trường khác nhau, các giải pháp tránh va chạm nhằm đảm bảo an toàn không thể áp dụng cho nhiều đối tượng điều khiển phương tiện khác nhau. Vì thế, việc kết hợp thêm các thông số tác động của môi trường vào quá trình đưa ra giải pháp xây dựng mô hình cảnh báo tránh va chạm này là điều được chúng tôi xác định khá quan trọng và sẽ mang lại hiệu quả tối ưu.

Giải pháp này về cơ bản sẽ xác định theo thời gian phản ứng $t$ của người điều khiển phương tiện. Dựa trên số liệu đã khảo sát trong nghiên cứu của tác giả $\mathrm{M}$. A. Hoque và cộng sự [7], chúng tôi xác định 05 yếu tố có tác động tích cực đến quá trình điều khiển phương tiện và sẽ tính toán trọng số cho các yếu tố này trong quá trình thiết lập mô hình cảnh báo tránh va chạm của giải pháp đưa ra. Cụ thể, các yếu tố này bao gồm: Độ tuổi của người điều khiển $X_{1}$ với trọng số $\omega_{1}$, thời gian kinh nghiệm điều khiển phương tiện $X_{2}$ với trọng số $\omega_{2}$, chỉ số sức khỏe $X_{3}$ với trọng số $\omega_{3}$, chỉ số tinh thần $X_{4}$ với trọng số $\omega_{4}$ và chỉ số thị lực $X_{5}$ với trọng số $\omega_{5}$.

Với các chỉ số lần lược có các giá trị như sau: $X_{3}=$ $(1,2,3,4,5) ; X_{4}=(1,2,3,4,5)$ và ma trận quyết định của từng yếu tố ảnh hưởng cùng thời gian phản ứng $T$ như sau:

$$
T=\left[\begin{array}{c}
X_{1} \\
X_{2} \\
\ldots \\
X_{m}
\end{array}\right]\left[\begin{array}{cccc}
X_{11} & X_{12} & \ldots & X_{1 n} \\
X_{21} & X_{22} & \ldots & X_{2 n} \\
\ldots & \ldots & \ldots & \ldots \\
X_{m 1} & X_{m 2} & \ldots & X_{m n}
\end{array}\right]
$$

và

$$
\begin{gathered}
P_{i j}=\frac{x_{i j}}{\sum_{i=1}^{m} x_{i j}} \\
E_{j}=-K \sum_{i=1}^{m} P_{i j} \ln \left(P_{i j}\right)
\end{gathered}
$$

Trong đó: $P_{i j}$ là tác động của thuộc tính thứ $i$ vào yếu tố $x_{i j}, E_{j} \in[0,1]$ là tổng tác động của quá trình vào chỉ số $X_{j}$ và hằng số $K=\frac{1}{\ln (m)}$.

Từ $(5),(6)$ và $(7)$ có thể thấy rằng vai trò tác động của mỗi lược đồ theo một thuộc tính nhất định có xu hướng giống nhau và giá trị $E$ tiến tới 1 . Trong trường hợp, khi tất cả yếu tố tác động đều bằng nhau thì vai trò thuộc tính mục tiêu trong quyết định có thể không xét đến, nghĩa là trọng số của chỉ số đang xét sẽ bằng 0 . Từ đó, có thể thấy rằng giá trị thuộc tính được xác định bởi sự khác biệt của tất cả các lược đồ. Do đó, mức độ tác động nhất quán $d_{j}$ của từng yếu tố trong thuộc tính thứ $\mathrm{j}$ được xác định như sau:

$$
d_{j}=1-E_{j}
$$

Khi đó, hệ số trọng số $\omega_{j}$ và khoảng thời gian đáp ứng $\tau_{d r}$ của hệ thống được tính như sau:

$$
\begin{gathered}
\omega_{j}=\frac{d_{j}}{\sum_{j=1}^{n} d_{j}} \\
Y_{i}=\sum_{i=1}^{5} X_{i} \omega_{i} \\
\tau_{d r}=1.2 \sqrt{\frac{75}{Y_{i}}}
\end{gathered}
$$

Thời gian đáp ứng của hệ thống là thời gian thực hiện của hệ thống phanh, bao gồm: thời gian đáp ứng của hệ thống phanh $t_{1}$, thời gian tác động của phanh $t_{2}$ và thời 
gian phanh liên tục $t_{3}$ [6]. Trong đó, thời gian phanh liên tục $t_{3}$ được giả định là giá trị không đổi để làm giảm tốc độ và được tính như sau:

$$
t_{3}=\frac{v}{3,6 * g * a * \mu}-\frac{t_{2}}{2}
$$

Với $v$ là vận tốc của xe chủ, $g=9.8 \mathrm{~m} / \mathrm{s}^{2}$ là gia tốc trọng trường trái đất, $\mu$ là hệ số trượt (là hệ số bám giữa bánh xe và mặt đường) và $a$ là chỉ số môi trường. Trong đó, các giá trị của chỉ số môi trường được đưa ra như sau: $a=1$ khi mặt đường khô, $a=0,5$ khi mặt đường có nước, $a=0,1$ khi mặt đường cát và $a=0,3$ khi mặt đường đồi núi.

Khoảng thời gian phanh liên tục sẽ thay đổi theo vận tốc của xe và hệ số trượt sẽ tương quan tỉ lệ nghịch với quãng đường phanh, do đó khoảng thời gian sẽ xảy ra va chạm $T T C_{a v}$ được tính như sau:

$$
T T C_{a v}(s)=\frac{D}{V_{\tau}}
$$

Trong đó: $D$ là khoảng cách giữa 2 xe và $V_{\tau}$ là vận tốc tương đối giữa 2 xe. Như vậy, ngưỡng thời gian an toàn $T T C_{C s}$ để tránh va chạm được tính là khoảng thời gian tối thiểu từ khi hệ thống phát ra tín hiệu cảnh báo nguy hiểm đến thời điểm mà người điều khiển phương tiện thực hiện thao tác tránh nguy hiểm, được tính như sau:

$$
T T C_{c s}(s)=t_{1}+t_{2}+t_{3}+\tau_{d r}
$$

Từ (10), (11), (12) và (14), ngưỡng thời gian an toàn $T T C_{c s}$ được tính như sau:

$$
T T C_{c s}(s)=t_{1}+\frac{t_{2}}{2}+\frac{v}{3,6 * g * a * \mu}+1.2 \sqrt{\frac{75}{\sum_{i=1}^{5} X_{i} \omega_{i}}}
$$

Trong đó: $X_{i}$ được xác định tùy vào giá trị thực tế của người điều khiển phương tiện. Vì dữ liệu thu nhận được từ môi trường gồm tốc độ và khoảng cách giữa các xe là tương đối, nên chúng ta cần chuyển đổi ngưỡng thời gian an toàn $T T C_{c s}$ thành khoảng cách xe tương ứng và chuẩn hóa các biến số. Điều này có thể làm giảm độ phức tạp về thời gian và không gian của thuật toán.

Từ đó, giá trị khoảng cách cảnh báo $D_{w}$ và khoảng cách phanh xe $D_{b}$ được tính như sau:

$$
\begin{gathered}
D_{w}=v_{\tau}\left(t_{1}+\frac{t_{2}}{2}+\frac{v}{3,6 * g * a * \mu}+1.2 \sqrt{\frac{75}{\sum_{i=1}^{5} X_{i} \omega_{i}}}\right) \\
D_{b}=v_{\tau}\left(\frac{t_{2}}{2}+\frac{v}{3,6 * g * a * \mu}\right)
\end{gathered}
$$

Với $V_{\tau}$ là vận tốc tương đối giữa 2 xe, $t_{1}$ là thời gian đáp ứng của hệ thống phanh, $t_{2}$ là thời gian tác động của phanh và $t_{3}$ là thời gian phanh liên tục, $g=9,8 \mathrm{~m} / \mathrm{s}^{2}$ là gia tốc trọng trường trái đất, $\mu$ là hệ số trượt, $a \in[1,0,5,0,1,0,3]$ là chỉ số môi trường, $X_{i}$ và $\omega_{i}$ là chỉ số tác động và trọng số

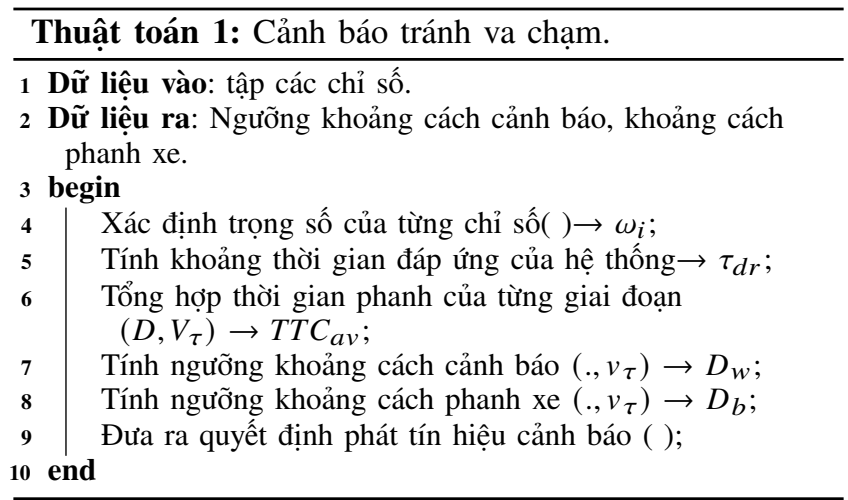

tương ứng. Như vậy, với việc chuyển đổi ngưỡng thời gian an toàn để tránh va chạm thành giá trị khoảng cách ngưỡng cảnh báo tương ứng và đưa ra tín hiệu cảnh báo cần thiết trong quá trình điều khiển chuyển động để tránh va chạm. Giải pháp cảnh báo tránh va chạm cho phương tiện tham gia giao thông được chúng tôi đưa ra trong nghiên cứu này sẽ được thực thi với các dữ liệu đầu vào là tốc độ thực tế, đặc điểm môi trường và đặc điểm người điều khiển phương tiện, với các bước cơ bản được trình bày trong thuật toán 1.

Biểu diễn bằng lưu đồ thuật toán:

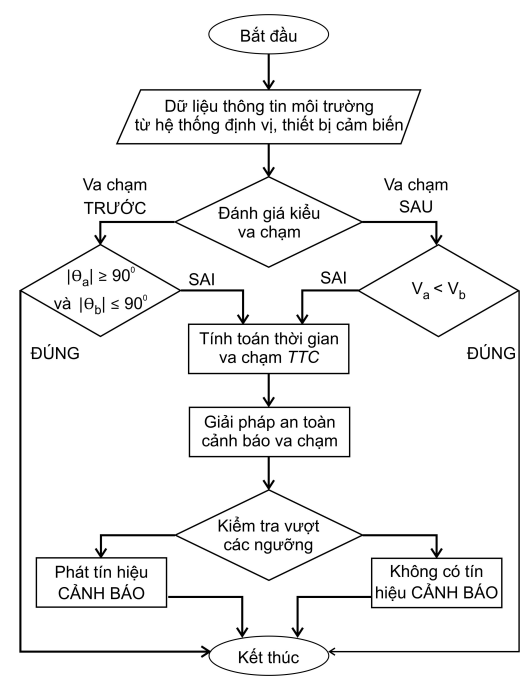

Hình 2. Lưu đồ thuật toán cảnh báo tránh va chạm

\section{III. ĐÁNH GIÁ VÀ KẾT LUẬN}

Để kiểm tra và đánh giá giải pháp đề xuất, chúng tôi tiến hành mô phỏng thực nghiệm các quy trình trong môi trường Matlap. Đồng thời, nhằm đảm bảo tính khách quan và độ tin cậy khi đánh giá, chúng tôi tiến hành mô phỏng với các kịch bản khác nhau, gồm kịch bản xảy ra va chạm trước và kịch bản xảy ra va chạm phía sau. Trong đó các tính toán sử 
dụng hệ đo lường SI với khoảng thời gian cập nhật của hệ thống $\tau=0,2 s$, các bộ thu nhận tín chuyển dữ liệu đến bộ điều khiển $\mathrm{I} / \mathrm{O}$ với chu kỳ thời gian mô phỏng là $0,05 \mathrm{~s}$. Quá trình mô phỏng để xác định giá trị khoảng cách cảnh báo an toàn được tiến hành với 03 tình huống: Tình huống thực hiện phanh khẩn cấp với phương tiện phía trước, tình huống vận tốc của xe phía trước nhỏ hơn xe chủ và tình huống cả 2 xe đều cùng vận tốc. Tham số trong mô phỏng này với vận tốc và gia tốc của các xe được quy định là bằng nhau, do đó giá trị vận tốc tương đối $v_{\tau}$ của các xe có 1 giá trị trong môi trường hoạt động và thời gian đáp ứng của hệ thống phanh $t_{1}=0,5 \mathrm{~s}$, thời gian tác động của phanh $t_{2}=0,4 \mathrm{~s}$, thời gian phanh liên tục $t_{3}=3 s$, hệ số trượt $\mu=0,7$, chỉ số đối tượng điều khiển phương tiện tạo tác động cho hệ thống được tiến hành 02 người, với các chỉ số như sau: $[O B J]_{a}=\left(X_{1}=30, X_{2}=5, X_{3}=7, X_{4}=1,8, X_{5}=5\right)$ và $[O B J]_{b}=\left(X_{1}=58, X_{2}=3, X_{3}=4, X_{4}=1,1, X_{5}=20\right)$.

Kết quả mô phỏng tiếp tục được đánh giá so sánh với các giải pháp áp dụng thuật toán khác gồm: thuật toán Mazda[13] và thuật toán Berkeley[13], quá trình đánh giá được tiến hành bằng cách so sánh khoảng cách thực hiện phanh an toàn với các vận tốc tương đối khác nhau.

Trong đó, thuật toán Mazda là thuật toán dựa trên phân tích động học để xác định khoảng cách giới hạn phanh. Tuy nhiên, khoảng cách được tính toán là khoảng cách để đảm bảo an toàn cho thao tác phanh, nên khoảng cách này được sử dụng làm khoảng cách giới hạn cảnh báo [11]. Khoảng cách cảnh báo $D_{w}$ được tính như sau:

$$
D_{w m a z d a}=\frac{1}{2}\left(\frac{v^{2}}{a_{1}}-\frac{\left(v-v_{0}\right)^{2}}{a_{2}}\right)+v t_{1}+v_{0} t_{2}+d_{0}
$$

Với $d_{0}$ là khoảng cách ban đầu, $\mathrm{v}$ là vận tốc của xe phía sau, $v_{0}$ là vận tốc tương đối giữa các xe, $t_{1}$ là thời gian trễ của hệ thống, $t_{2}$ là thời gian phản ứng của người điều khiển, $a_{1}$ là vận độ giảm cực đại do thao tác phanh của xe phía sau và $a_{2}$ là vận độ giảm cực đại do thao tác phanh của xe phía trước.

Và thuật toán Berkeley là là thuật toán được cải tiến dựa trên thuật toán Mazda nhằm tạo ra khoảng cách cảnh báo an toàn hơn, phương trình khoảng cách cảnh báo $D_{\text {wberkeley }}$ được xây dựng như sau:

$$
D_{w m a z d a}=\frac{1}{2}\left(\frac{v^{2}}{a}-\frac{\left(v-v_{0}\right)^{2}}{a}\right)+v\left(t_{1}+t_{2}\right)+d_{0}
$$

Với $d_{0}$ là khoảng cách ban đầu, $v$ là vận tốc của xe phía sau, $v_{0}$ là vận tốc tương đối giữa các xe, $t_{1}$ là thời gian trễ của hệ thống, $t_{2}$ là thời gian phản ứng của người điều khiển, $a$ là vận độ giảm cực đại do thao tác phanh của xe.

$$
\begin{gathered}
\xi=\frac{d-d_{\xi}}{d_{\xi}-d_{b r}} \\
d_{b r}=v_{0}\left(t_{1}+t_{2}\right)+\frac{1}{2} a\left(t_{1}+t_{2}\right)^{2}
\end{gathered}
$$

Với $d$ là khoảng cách hiện tại giữa 2 xe, $d_{b r}$ là khoảng cách giới hạn phanh.

\section{Kết quả mô phỏng cảnh báo va chạm phía sau}

Quá trình thực hiện mô phỏng trong các điều kiện môi trường khác nhau, với 04 chỉ số môi trường được thiết lập cho dữ liệu đầu vào gồm $a \in[1,0,5,0,1,0,3]$ tương ứng với môi trường mặt đường khô, mặt đường có nước, mặt đường cát và mặt đường đồi núi.

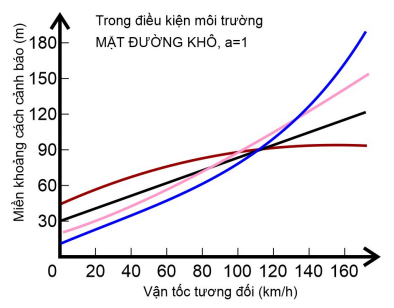

(A)

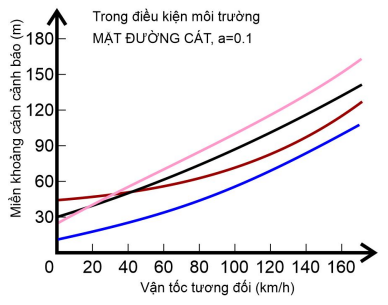

(C)

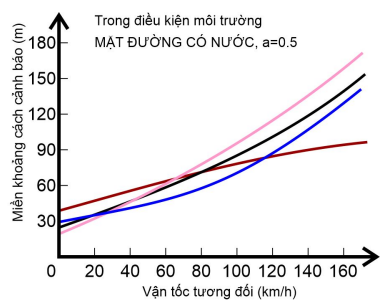

(B)

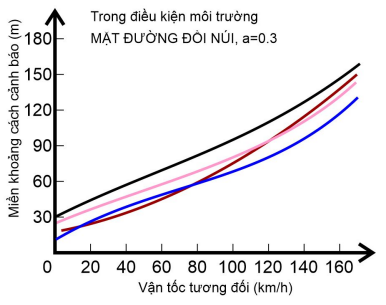

(D)

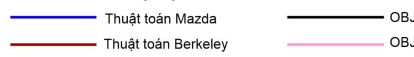

Hình 3. Kết quả mô phỏng cảnh báo va chạm sau

Kết quả số liệu mô phỏng cho thấy với tốc độ di chuyển khác nhau thì trong điều kiện môi trường tốt (mặt đường khô) khoảng cách cảnh báo của $O B J_{a}$ (có chỉ số độ tuổi ít) ngắn hơn so với $O B J_{b}$ (có chỉ số độ tuổi nhiều), nghĩa là người trẻ tuổi sẽ có phản xạ nhanh hơn khi thay đổi tốc độ so với người lớn tuổi trong điều kiện bình thường và người có chỉ số tuổi lớn thì thao tác thận trọng hơn. Nhưng trong điều kiện mặt đường có nước, mặt đường cát thì khoảng cách cảnh báo của $O B J_{a}$ tăng lên rất nhiều và cũng tương đương với khoảng cách cảnh báo của $O B J_{b}$, nghĩa là với chỉ số kinh nghiệm điều khiển còn ít thì khoảng cách dự báo cần thay đổi cho phù hợp với điều kiện thời tiết không thuận lợi. Như vậy, qua kết quả mô phỏng chúng ta có thể thấy được tác động của điều kiện môi trường làm thay đổi quá trình phát tín hiệu cảnh báo tránh va chạm, và tùy thuộc tập chỉ số đầu vào của đối tượng điều khiển phương tiện mà khoảng cách cảnh báo cũng thay đổi tương ứng.

\section{Kết quả mô phỏng cảnh báo va chạm trực diện}

Quá trình thực hiện mô phỏng trong các điều kiện môi trường khác nhau, với 04 chỉ số môi trường được thiết lập cho dữ liệu đầu vào gồm $a \in[1,0,5,0,1,0,3]$. 


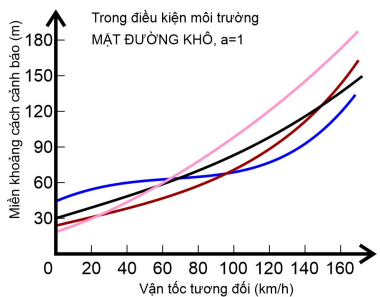

(A)

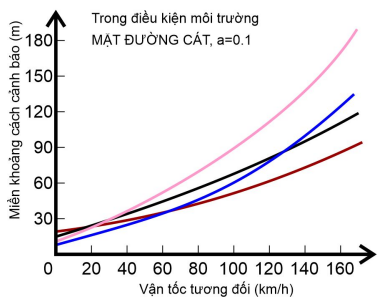

(C)

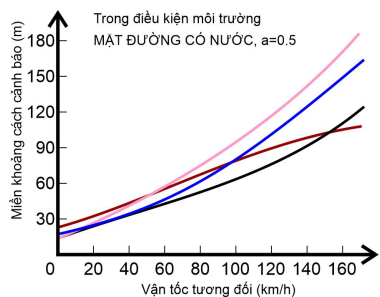

(B)

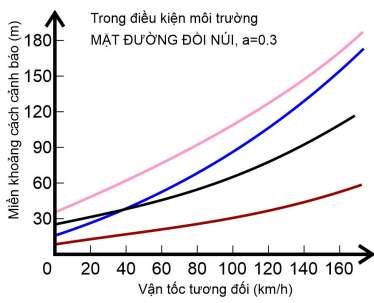

(D)

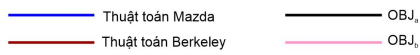

Hình 4. Kết quả mô phỏng cảnh báo va chạm trực diện

Kết quả mô phỏng cho thấy, khoảng cách cảnh báo tránh va chạm của giải pháp áp dụng thuật toán Berkeley ít thay đổi theo vận tốc tương đối. Ngoài ra, khi vận tốc tương đối cao thì khoảng cách cảnh báo ngắn nên không kịp thời và khi tốc độ tương đối thấp dễ gây ra cảnh báo sai, từ đó dẫn đến điều kiện an toàn trong cảnh báo nguy hiểm không đảm bảo. Trong mô phỏng tránh va chạm trực diện này thì khoảng cách cảnh báo khi áp dụng giải pháp đề xuất với các chỉ số đầu vào của $O B J_{a}, O B J_{b}$ thì khoảng cách cảnh báo tránh va chạm nhạy cảm hơn với sự thay đổi tốc độ tương đối, nên có thể đảm bảo an toàn cho các phương tiện với các tốc độ tương đối khác nhau. Trong điều kiện các chỉ số môi trường hoạt động khác nhau, với vận tốc tương đối chậm thì khoảng cách dự báo của giải pháp đề xuất gần bằng với khoảng cách dự báo được thực hiện bởi thuật toán Berkeley, nhưng khi vận tốc tương đối tăng thì khoảng cách dự báo giữa 2 giải pháp cũng tăng tỷ lệ thuận và mức độ gia tăng khoảng cách cảnh báo của giải pháp đề xuất tăng nhanh hơn so với giải pháp áp dụng thuật toán Berkeley.

Còn với giải pháp áp dụng thuật toán Mazda có thời gian cảnh báo tương đối chậm trong các điều kiện môi trường khác nhau, khoảng cách cảnh báo ngắn và chậm, điều này vẫn chưa chiếm được ưu điểm so với giải pháp được đề xuất trong nghiên cứu này.

\section{Kết luận}

Như vậy với giải pháp kết hợp giữa thời gian tránh va chạm và các chỉ số liên quan về môi trường hoạt động, chỉ số liên quan đến đối tượng điều khiển phương tiện, giải pháp đề xuất đã giải quyết bài toán cảnh báo tránh va chạm dưới góc độ xét các mối quan hệ giữa vị trí các đối tượng, các yếu tố tác động của môi trường như hệ số trượt, tầm nhìn để tạo ra các khoảng thời gian cảnh báo khác nhau, với kết quả mô phỏng ban đầu, đã cho thấy khả năng thích nghi của giải pháp đề xuất có thể áp dụng với nhiều chỉ sô đầu vào tùy thuộc vào môi trường hoạt động, đã giảm tỷ lệ cảnh báo sai và cảnh báo thiếu so với các giải pháp áp dụng thuật toán Berkeley và Mazda. Thuật toán đề xuất đã chứng minh tính hợp lý, khả thi và có thể cải thiện hiện quả an toàn giao thông thông qua thực nghiệm.

Ý tưởng chính của giải pháp kỹ thuật này sẽ hỗ trợ cho việc thiêt kế xe một điểm dừng an toàn, cho dù việc điều khiển xe hiện tại là như thế nào. Trong tương lai, để tăng độ tin cậy của giải pháp này thì những thiết lập đã được thực nghiệm bằng mô phỏng sẽ được chuyển sang môi trường thực với xe thực nghiệm được trang bị đầy đủ các cảm biến, đồng thời khi thực nghiệm trên thực tế sẽ bổ sung một số yếu tố phân tích tính ổn định của hệ thống sao cho hành vi tham gia giao thông của các đối tượng được dự báo chính xác hơn. Việc triển khai rộng rãi giải pháp này cho các xe bán tự hành trong các hệ thống điều khiển phương tiện sẽ có thể giảm thiểu được số lượng lớn các thiệt hại cũng như tạo ra một kế hoạch chuyển động an toàn cho tương lai.

\section{TÀI LIỆU THAM KHẢO}

[1] A. Thakur and R. Malekian (2019), "Internet of vehicles communication technologies for traffic management and road safety applications" Wireless Personal Communications, vol. 109, no. 1, pp. 31-49, DOI:10.1007/s11277-01906548-y.

[2] E. Awad, S. Dsouza, R. Kim et al. (2018), "The moral machine experiment", Nature, vol. 563, no. 7729, pp. 59-64, DOI: $10.1038 / \mathrm{s} 41586-018-0637-6$.

[3] H. Peng, L. Le Liang, X. Shen, et al. (2019), "Vehicular communications: a network layer perspective", IEEE Transactions On Vehicular Technology, vol. 68, no. 2, pp. 1064-1078, DOI: 10.1109/TVT.2017.2750903.

[4] H. Zhou, N. Cheng, J. Wang et al (2019), “Toward dynamic link utilization for efficient ve-hicular edge content distribution”, IEEE Transactions On Vehicular Technology,vol. 68, no. 9, pp. 8301-8313, DOI: 10.1109/TVT.2019.2921444.

[5] L. Yang, J. Wang, C. Gao et al (2018), "A crisis information propagation model based on a competitive relation", Journal of Ambient Intelligence and Humanized Computing,, vol. 10, no. 8, pp. 2999-3009, DOI: 10.1007/s12652-018-0744-0.

[6] L. Jin, B. Arem, S. Yang (2019), "Performance analysis and boost for a MAC protocol in vehicular networks", IEEE Transactions on Vehicular Technology,vol. 68, no. 9, pp. 8721-8728, DOI: 10.1109/TVT.2019.2927228.

[7] M. A. Hoque, X. Hong, M. S. Ahmed (2019), "Parallel closed-loop connected vehicle simu-lator for largescale transportation network management: challenges, issues, and solution ap-proaches", IEEE Intelligent Transportation Systems Magazine, vol. 11, no. 4, pp. 62-77, DOI: 10.1109/MITS.2018.2879163.

[8] N. Jiang, F. Tian, J. Li et al. (2019), "MAN: mutual attention neural networks model for as-pect-level sentiment classification in SIot", IEEE Internet of Things Journal,vol. 15, no. 3, pp. 1054-1065, DOI: 10.1109/JIOT.2020.2963927. 
[9] R. Molina-Masegosa, J. Gozalvez (2017), "LTE-V for sidelink 5G V2X vehicular commu-nications: a new 5G technology for short-range vehicle-to-everything communications", IEEE Vehicular Technology Magazine, vol. 12, no. 4, pp. 30-39, DOI: 10.1109/MVT.2017.2752798.

[10] S. Sharma, B. Kaushik (2019), "A survey on internet of vehicles: applications, security is-sues and solutions" , Vehicular Communications, vol. 20, no. 5, pp. 725-729, DOI: 10.1016/j.vehcom.2019.100182.

[11] S. Jeong, O. Simeone, J. Kang (2018), "Mobile edge computing via a UAV mounted cloud let: optimization of bit allocation and path planning", IEEE Transactions On Vehicular Technology, vol. 67, no. 3, pp. 2049-2063, DOI: 10.1109/TVT.2017.2706308.

[12] X. Pei (2012), "Safe distance model and obstacle detection algorithms for A collision warn-ing and collision avoidance system”, Automotive Safety and Energy, vol. 3, no. 1, pp. 26-33, DOI: 10.3969/j.issn.1674-8484.2012.01.004.

[13] Ararat, Oncu and Kural, Emre and Guvenc, Bilin Aksum."Development of a collision warning system for adaptive cruise control vehicles using a comparison analysis of recent algorithms", 2006 IEEE Intelligent Vehicles Symposium, pp. 194-199, 2006

\section{SƠ LƯƠC VỀ TÁC GIẢ}

\section{Quách Hải Thọ}

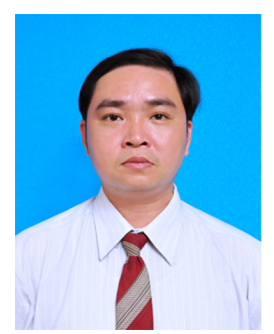

Sinh năm 1978, tốt nghiệp cử nhân ngành tin học (2000) và thạc sĩ chuyên ngành Khoa học máy tính (2012) tại trường Đại học Khoa học, Đại học Huế. Hiện đang làm NCS ngành Hệ thống thông tin tại trường Đại học Sư phạm, Đại học Đà nẵng. Đơn vị công tác: Phòng Đào tạo, BĐCL\& CTSV, trường Đại học Nghệ thuật, Đại học Huế. Lĩnh vực nghiên cứu: Đồ họa máy tính, hoc máy.

Email: qhaitho@hueuni.edu.vn

Tel: 0913439816

\section{Huỳnh Công Pháp}

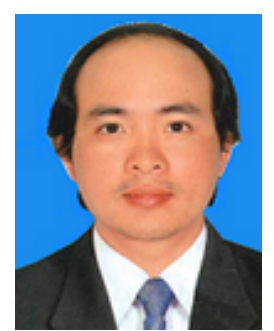

Sinh năm 1977, đã nhận bằng Tiến sĩ (2010) ngành Công nghệ thông tin tại Đại học Bách Khoa Grenoble-CH Pháp \& Viện CNTT Quốc gia NII-Tokyo-Nhật. Hiện là Hiệu trưởng trường Đại học Công nghệ thông tin và Truyền thông Việt - Hàn, Đại học Đà Nẵng. Lĩnh vực nghiên cứu: Mang máy tính, Lập trình mạng, Web ngữ nghĩa. Email: hcphap@vku.udu.vn

\section{Phạm Anh Phương}

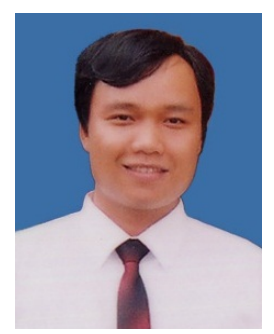

Sinh năm 1974, đã tốt nghiệp đại học (1996) ngành Toán - Tin học tại trường Đại học Sư phạm, Đại học Huế, tốt nghiệp Thac sĩ (2001) chuyên ngành Khoa học Máy tính tại Đại học Bách Khoa Hà Nội và nhận bằng Tiến sĩ (2010) chuyên ngành - Bảo đảm toán học cho máy tính và hệ thống tính toán tại Viện Công nghệ Thông tin, Hà Nội. Hiện là Phó Trưởng Khoa Khoa Tin hoc, Trường ĐH Sư Pham - Đai học Đà Nẵng. Lĩnh vực nghiên cứu: Kỹ thuật lập trình; Đồ họa máy tính, Xử lý ảnh, lý thuyết nhận dạng.

Email: paphuong@ued.udn.vn 